\title{
Genome-Wide Association Studies on Attention Deficit Hyperactivity
} Disorder

\author{
Kathryn Ashmore ${ }^{1}$ and Feng Cheng ${ }^{2 *}$ \\ ${ }^{1}$ Department of Chemistry, University of South Florida, USA \\ ${ }^{2}$ Department of Pharmaceutical Science, College of Pharmacy, University of South Florida, USA
}

\begin{abstract}
A Genome-Wide Association Study (GWAS) is an approach to identify common genetic variations associated with a disease. It is widely used to determine the genetic basis of diseases. In this paper, the applications of GWAS on Attention Deficit Hyperactivity Disorder (ADHD) were reviewed.
\end{abstract}

\section{Attention-Deficit Hyperactivity Disorder}

ADHD is apsychiatric disease in children and adults characterized by inattention, impulsivity, and hyperactivity. It is estimated by the US Center for Disease Control (CDC) that three to seven percent of school aged children are diagnosed with ADHD. ADHD is a serious issue in classrooms and families because it affects not only the child, but his or her family, classmates, and teachers. Furthermore, children with the disorder often struggle in school due to their inability to focus for long periods of time. There is currently no efficient or cost-effective way to treat or diagnose this disorder because the mechanisms underlying ADHD remain unclear. ADHD is considered to be a highly heritable disorder from the previous family-based study [1]. The heritability of this disease is about 0.8 [2]. However, it is difficult to identify genes or susceptibility loci associated with the disorder. Therefore, new research and clinical studies are imperative and invaluable.

\section{Genome-Wide Association Studies}

GWAS have been conducted on ADHD to detect common genetic variants, including Single-Nucleotide Polymorphism (SNP) and Copy Number Variation (CNV), to see if there are any genes involved in ADHD. GWAS has the potential to find novel disease-related genes because it investigates all human genes in the whole genome instead of specific genes or chromosome regions. GWAS is therefore referred to as a non-candidate-gene-driven study. In the NIH GWAS database (http://www.genome.gov/26525384), 8,175 SNPs from 1,476 GWAS publications had reached genome-wide significance $(\mathrm{P}$-value $\leq 5 \times 10$ ${ }^{8}$ ) for over 200 diseases by January 2013.In this paper, we will review some progress in GWAS on ADHD.

GWAS has two experimental designs based on the sample collection: unrelated case-control design and family-based design (trio design). In the former design, patients (cases) and unrelated participants without the disease (controls) are selected. A linear regression is performed to test associations of SNPs and disease status. If a SNP is more frequent in patients than in controls, the SNP might be considered to be "associated" with the disease. The unrelated casecontrol design is simple and widely used. The disadvantage of this design is the population stratification bias (or confounding by race/ ethnicity) that could bring false positives. In the family-based design, ADHD patients and their parents (trio members) are chosen. The frequency of an allele transmitted from heterozygous parents to affected offspring is calculated. The population stratification bias can be bypassed in the family-based design because family members have a similar genetic background and environmental effects. The limitation of the family-based design is the difficulty of collecting large numbers of family samples.

\section{Applications of GWAS on ADHD}

Recently, large scale genome-wide association studies [2-10] have been applied to identify novel ADHD risk genes using either unrelated case-control or family-based design (Table 1). An unrelated case-control GWAS was conducted on adult ADHD patients using Affymetrix Human Mapping 500K SNP microarray by Lesch et al. [3]. Samples from 343 adult ADHD patients and 250 controls of German origin were investigated in this study. There was no SNP that reached stringent genome-wide significance. However, among the gene list derived from the 30 highest ranking SNPs, genes (such as CDH13, ASTN2, and CTNNA2) identified by the GWAS in other neuro developmental and psychiatric disorders were also shown. The first case-control GWAS was performed on children and adolescents by Neale et al. [4] . DNA samples from 896 young ADHD patients and 2,455 controls of European origin were collected by the International Multicenter ADHD Genetics (IMAGE) project from Europe and the USA. 1,033,244 SNPs were analyzed in this study and the best SNP was rs10823973 located in PRKGlat chromosome 10 and its p-value was $6.32 \times 10^{-7}$. Hinney et al. [2] performed an unrelated case-control on 495 German ADHD children/adolescents and 1,300 controls. 11,917 $\mathrm{X}$-chromosomal and 487,484 autosomal SNPs were investigated and the lowest $p$-value was $8.37 \times 10^{-7}$ at the SNP rs2556378. Although no genome-wide significance ( $\mathrm{p}$-value $\leq 5 \times 10^{-8}$ ) was detected, gene, GRM5 identified from the top 30 SNPs, has been reported as a candidate gene by previous studies. Stergiakouli et al. [5] performed a GWAS based on 727 Caucasian children with ADHD and 5,081 controls from the United Kingdom and Ireland. 518,511 SNPs was analyzed and the lowest p-value was $6.38 \times 10^{-6}$ at rs11698703. CHRNA7, one of the nicotinic acetylcholine receptors, was believed to be a promising candidate gene. CNV analysis showed CHRNA7 had six large duplications in ADHD patients and none in the control people. The smallest p-value of SNP in this gene was 0.0002 .

Several family-based GWAS studies also have been conducted

*Corresponding author: Feng Cheng, Department of Pharmaceutical Science, College of Pharmacy, University of South Florida, USA, Tel: 813-974-4288; Fax: 813-905-9890; E-mail: fcheng1@health.usf.edu

Received January 25, 2013; Accepted February 11, 2013; Published February 14,2013

Citation: Ashmore K, Cheng F (2013) Genome-Wide Association Studies on Attention Deficit Hyperactivity Disorder. Clin Exp Pharmacol 3: 119 doi:10.4172/2161-1459.1000119

Copyright: (c 2013 Ashmore K, et al. This is an open-access article distributed under the terms of the Creative Commons Attribution License, which permits unrestricted use, distribution, and reproduction in any medium, provided the original author and source are credited. 
Citation: Ashmore K, Cheng F (2013) Genome-Wide Association Studies on Attention Deficit Hyperactivity Disorder. Clin Exp Pharmacol 3: 119. doi:10.4172/2161-1459.1000119

Page 2 of 2

\begin{tabular}{|c|c|c|c|}
\hline Study & Type & Sample Size & Promising Candidate Genes \\
\hline Lesch [3] & Case-control & $343 / 250$ & CDH13, ASTN2, CTNNA2 and KALRN \\
\hline Neale [4] & Case-control & $896 / 2,455$ & PRKG1, FLNC, TCERG1L, PPM1H, NXPH1, PPM1H, CDH13, HK1, and HKDC1 \\
\hline Hinney [2] & Case-control & $495 / 1,300$ & GRM5 \\
\hline Stergiakouli [5] & Case-control & $727 / 5,081$ & CHRNA7 \\
\hline Neale [6] & Family & 909 trios & XKR4, and FAM190A \\
\hline Lasky-Su [7] & Family & 930 trios & SLC9A9 \\
\hline Mick [8] & Family & 735 trios & SLC9A9 \\
\hline Neale [9] & Combined & 2,064 trios and $896 / 2,455$ & CHMP7, TNFRSF10D, TNFRSF10A, and LOXL2 \\
\hline
\end{tabular}

Table 1: Large scale genome-wide association studies on attention-deficit hyperactivity disorder.

to identify ADHD-related genes. Neale et al. analyzed about 438,784 SNPs in 909parent-offspring trios of European descent. Similar to the unrelated case-control studies, no SNPs showed genome-wide significance. Lantieri et al. [10] validated the 36 top genes of Neale's family-based study using 415 independent trios in the USA. They found two SNPs were significant at $\alpha=0.05$ in this independent test set. These significant SNPs are located at the coding regions of XKR4 and FAM190A. Lasky-Su et al. [7] investigated 429,981 autosomal SNPs in 930 European trios. The lowest p-value was $1.02 \times 10^{-7}$ at rs9451437 in chromosome 6 . There are 4 SNPs with p-values $<10^{-5}$ located in intron regions of gene ADAMTS2. They investigated some SNPs among previous-reported ADHD genes and found SLC9A9might be a promising candidate gene because there were 6 associations with p-value $<0.05$ at 6 distinct regions of this gene. Mick et al. [8] also performed a family-based GWAS on $835,136 \mathrm{SNP}$ in 735 ADHD trios in the USA. The lowest p-value was $6.7 \times 10^{-7}$. One top SNP, rs9810857, was located in gene SLC9A9, which is consistent with Lasky-Su's hypothesis. Additionally, Neale et al. [9] also combined familybased and case-control data from four international ADHD research projects together and performed a meta-analysis. This study included 2,064 trios, 896 cases, and 2,455 controls. However, no genome-wide significant associations were found despite this larger scale attempt.

In summary, neither unrelated case-control GWAS nor familybased GWAS led to the identification of significant ADHD-related SNPs at the genome-wide level. There were some explanations for the negative GWAS findings [5,6,9,11]. First, ADHD might be a multifactorial disorder caused by some common genetic variants with small effect sizes. These variants could not be well detected with genome-wide statistical confidence at current sample sizes. Second, although combining samples from different GWAS studies could increase the sample size, inconsistent genotyping platforms and ADHD evaluation criteria might eliminate positive findings. Thirdly, ADHD might be caused by rare (or low frequency) alleles that cannot be well detected by SNP microarrays. With the development of the next generation sequencing, whole genome or exome sequencing will provide an opportunity to capture these rare variants. Fourth, there might be multiple ADHD subtypes which have different genetic mechanisms. The genetic heterogeneity might dilute the effects of real ADHD SNPs.

\section{Prospective}

Although recently published GWAS data have failed to identify genome-wide significant ADHD-related SNPs and genes, they provided some promising candidate genes and chromosome loci for further validation. These GWAS data sets could be pooled together for larger-scale meta-analyses. Additionally, multi-site collaborations and standardized protocols are necessary for collecting larger ADHD patient data sets in the future.

\section{Acknowledgements}

This work was supported by the start-up grant from the College of Pharmacy at the University of South Florida.

\section{Reference}

1. Faraone SV, Perlis RH, Doyle AE, Smoller JW, Goralnick JJ, et al. (2005) Molecular genetics of attention-deficit/hyperactivity disorder. Biol Psychiatry 57: 1313-1323.

2. Hinney A, Scherag A, Jarick I, Albayrak Ö, Pütter C, et al. (2011) Genomewide association study in German patients with attention deficit/hyperactivity disorder. Am J Med Genet B Neuropsychiatr Genet 156B: 888-897.

3. Lesch KP, Timmesfeld N, Renner TJ, Halperin R, Röser C, et al. (2008) Molecular genetics of adult ADHD: converging evidence from genome-wide association and extended pedigree linkage studies. J Neural Transm 115: 1573-1585.

4. Neale BM, Medland S, Ripke S, Anney RJ, Asherson P, et al. (2010) Casecontrol genome-wide association study of attention-deficit/hyperactivity disorder. J Am Acad Child Adolesc Psychiatry 49: 906-920.

5. Stergiakouli E, Hamshere M, Holmans P, Langley K, Zaharieva I, et al. (2012) Investigating the contribution of common genetic variants to the risk and pathogenesis of ADHD. Am J Psychiatry 169: 186-194.

6. Neale BM, Lasky-Su J, Anney R, Franke B, Zhou K, et al. (2008) Genome-wide association scan of attention deficit hyperactivity disorder. Am J Med Genet B Neuropsychiatr Genet 147B: 1337-1344.

7. Lasky-Su J, Anney RJ, Neale BM, Franke B, Zhou K, et al. (2008) Genomewide association scan of the time to onset of attention deficit hyperactivity disorder. Am J Med Genet B Neuropsychiatr Genet 147B: 1355-1358.

8. Mick E, Todorov A, Smalley S, Hu X, Loo S, et al. (2010) Family-based genome-wide association scan of attention-deficit/hyperactivity disorder. J Am Acad Child Adolesc Psychiatry 49: 898-905.

9. Neale BM, Medland SE, Ripke S, Asherson P, Franke B, et al. (2010) Metaanalysis of genome-wide association studies of attention-deficit/hyperactivity disorder. J Am Acad Child Adolesc Psychiatry 49: 884-897.

10. Lantieri F, Glessner JT, Hakonarson H, Elia J, Devoto M (2010) Analysis of GWAS top hits in ADHD suggests association to two polymorphisms located in genes expressed in the cerebellum. Am J Med Genet B Neuropsychiatr Genet 153B: 1127-1133.

11. Franke B, Neale BM, Faraone SV (2009) Genome-wide association studies in ADHD. Hum Genet 126: 13-50. 